\title{
Craniomaxillofacial osteosarcoma - The role of surgical margins
}

\author{
R. Kontio a , J. Hagström ${ }^{\text {b }}$, P. Lindholm ${ }^{\text {e }}$, T. Böhling ${ }^{\text {b }}$, M. Sampo ${ }^{\text {b }}$, K. Mesimäki ${ }^{\text {a }}$, \\ K. Saarilahti ${ }^{c}$, P. Koivunen ${ }^{f}$, A.A. Mäkitie ${ }^{\text {d, g, h, * }}$ \\ a Department of Oral and Maxillofacial Surgery - Head and Neck Surgery, University of Helsinki and HUS Helsinki University Hospital, Helsinki, Finland \\ ${ }^{\mathrm{b}}$ Department of Pathology - Head and Neck Surgery, University of Helsinki and HUS Helsinki University Hospital, Helsinki, Finland \\ ${ }^{c}$ Department of Oncology - Head and Neck Surgery, University of Helsinki and HUS Helsinki University Hospital, Helsinki, Finland \\ d Department of Otorhinolaryngology - Head and Neck Surgery, University of Helsinki and HUS Helsinki University Hospital, Helsinki, Finland \\ e Department of Oncology, Turku University Hospital, Turku, Finland \\ f Department of Otorhinolaryngology - Head and Neck Surgery, Oulu University Hospital, Oulu, Finland \\ ${ }^{\mathrm{g}}$ Division of Ear, Nose and Throat Diseases, Department of Clinical Sciences, Intervention and Technology, Karolinska Institutet, Karolinska University \\ Hospital, Stockholm, Sweden \\ ${ }^{\mathrm{h}}$ Research Programme in Systems Oncology, Faculty of Medicine, University of Helsinki, Helsinki, Finland
}

\section{A R T I C L E I N F O}

Article history:

Paper received 18 December 2018

Accepted 18 March 2019

Available online $\mathrm{xxx}$

\section{Keywords:}

Osteosarcoma

Head and neck

Maxillofacial

Surgery

Radiotherapy

Survival

\begin{abstract}
A B S T R A C T
Background: Osteosarcomas (OS) in the craniomaxillofacial (CMF) region are typically diagnosed at later age than long-bone OS, but they are reported to have better 5-year survival. Curative treatment warrants wide surgical resection, which is often not possible in the CMF region. The purpose of this article is to present a nationwide series of CMF in Finland to discuss the role of surgery.

Patients and methods: All 21 CMF OS patients managed in Finland from 1992 to 2009 were included. The mean age was 40 years (range 15-72). Data on patient and tumor characteristics, treatment modalities, and survival were recorded. All patients had a minimum follow-up of 5 years or until death.

Results: OS was evenly represented in the mandible and maxillary bones, which together constituted 76\% of all sites. Surgery with curative intent was carried out in 20 patients. Clear margins were achieved in only five cases. Eight (40\%) of these 20 patients died due to OS, and their average survival time was 1.3 years. Seven (35\%) out of the 20 patients received radiotherapy due to close/intralesional surgical margins, and four of them did not develop recurrences during the follow-up.

Conclusions: The results suggest that postoperative radiotherapy may alter the prognosis in CMF OS, particularly in cases with close or intralesional margins. This may increase the survival rates achieved by prompt action in performing radical surgery.
\end{abstract}

(c) 2019 European Association for Cranio-Maxillo-Facial Surgery. Published by Elsevier Ltd. All rights reserved.

\section{Introduction}

Osteosarcoma (OS) of the craniomaxillofacial (CMF) bones is a rare malignant disease, comprising less than $1 \%$ of all malignant tumors in this region. However, based on published reports, approximately $5-10 \%$ of all OSs are situated in CMF region, with most found in the extremities, the pelvis, and axial skeleton (August et al., 1997; Fernandes et al., 2007). The tumor has mesenchymal origin and, although there is a definite histopathological similarity, it seems that there are certain differences in the

\footnotetext{
* Corresponding author. Department of Otorhinolaryngology - Head and Neck Surgery, Helsinki University Hospital, P.O. Box 263, FI-00029 HUS, Helsinki, Finland. E-mail address: antti.makitie@hus.fi (A.A. Mäkitie).
}

pattern of clinical behaviour between OSs of the extremities and those in the CMF area.

Most high-grade OSs occur in children, adolescents, or young adults (Chidzonga and Mahomva, 2007; Laskar et al., 2008). The mean age at presentation of patients with a CMF disease is about 10 years higher than that of those with long-bone OS. The rate of metastasis seems to be lower in the patient population having the lesion in CMF bones (August et al., 1997). Histopathological grade of OS is an important prognostic factor. Low-grade OSs can be managed by local therapy alone, while high-grade OSs warrant a combined treatment approach of chemotherapy and surgery. The overall survival rate seems to be better when the disease is localized in CMF bones (Yamaguchi and Nagasawa, 2004; Jasnau et al., 2008; Ketabchi et al., 2011). 
Reports focusing on OS of CMF bones are sparse and there are many uncertainties relating to the behaviour and treatment of this subgroup. Resectability depends on the size and location of the tumor in these anatomically and functionally challenging sites (Laskar et al., 2008). The primary treatment of OS is radical surgical removal with clear margins, which in long bones typically means more than $3 \mathrm{~cm}$. This approach is difficult to achieve in the CMF region due to certain vital structures, especially in the maxillary and zygomatic-orbital area (Sumida et al., 2015). Furthermore, the guidelines regarding additional treatment remain unclear if the resection is intralesional or with close margins (Thiele et al., 2008, Ketabchi et al., 2011). While some reports recommend neoadjuvant chemotherapy to improve survival rate, others state the opposite (Ketabchi et al., 2011). Also, the role of postoperative radiotherapy remains controversial, although it has been suggested for increasing local control after surgery with positive margins (Laskar et al., 2008; Guadagnolo et al., 2009; Lee et al., 2015).

Management of CMF OS is centred on the five university hospitals in Finland, which has a population of 5.5 million. Our study investigated nationwide data on these tumors with the primary aim to find out the treatment modalities used, and subsequent long-term survival in this population-based series. The secondary aim was to discuss the role of radical surgery in the management of CMF OS.

\section{Material and methods}

Retrospective data on all patients who had been diagnosed and managed for primary OS of the skull or maxillofacial region at the five university hospitals in Finland during the years 1992-2009 were collected from hospital records and pathology registries. All paraffin specimens were investigated by two pathologists ( $\mathrm{JH}$ and TB) to verify the histology. The study group comprised 21 patients with a mean age of 40 years (median 41; range 15-72). The ICD codes and search terms used were as follows: C41.0 (malignant tumor of skull or maxillofacial bones), C41.03 (OS of maxillary bone), C41.07 (OS of skull or maxillofacial bones), C41.09 (malignant tumor of skull or maxillofacial bones with unclear histology), C41.1 (malignant tumor of mandible), C41.13 (OS of mandible), and C41.19 (malignant tumor of mandible with unclear histology).

Data on sex, age, symptoms at presentation, tumor histology, site and extension, treatment, and outcome were collected. If the tumor had infiltrated into adjacent tissue, the original localization of the tumor was determined by the direction of tumor growth. The completeness of surgery was evaluated from pathology reports and divided into three categories: intralesional, close margins, and clear margins. Disease-free survival (DFS) was calculated from the end of treatment to the detection of cancer recurrence or death caused by OS. The dates and causes of death were provided by Statistics Finland. All patients had a minimum follow-up of 5 years or until death. The average follow-up time was 6.1 years (median 4.7; range $0.3-21.2$ ).

An institutional research approval was granted for the study at each participating hospital.

\section{Results}

Patient and tumor demographics are presented in Table 1. OS in the mandible and maxillary bones were evenly represented in this series, and together constituted $76 \%$ of all involved sites.

Five (24\%) out of the 21 patients had had a previous malignancy before the onset of OS and had thus received radiotherapy for the head and neck area (Table 2). OS originated in all these five patients in an area that had been included in their previous radiotherapy field $1,6,7,13$, or 19 years earlier, respectively.
Table 1

Demographic data for 21 patients with craniomaxillofacial OS.

\begin{tabular}{lll}
\hline Demographic variable & $n$ & $\%$ \\
\hline Age & & \\
$\quad<30$ years & 8 & 38 \\
$\quad \geq 30$ years & 13 & 62 \\
Sex & & \\
$\quad$ Male & 14 & 67 \\
Female & 7 & 33 \\
Follow-up time & & \\
$<4$ years & 10 & 48 \\
$4-8$ years & 4 & 19 \\
$8-12$ years & 4 & 19 \\
$>12$ years & 3 & 14 \\
Localization & & 38 \\
Mandibular bone & 8 & 38 \\
Maxillary bone & 8 & 5 \\
Zygomatic bone & 1 & 14 \\
Frontal, ethmoid bone & 3 & 5 \\
$\quad$ Temporal bone & 1 & \\
\hline
\end{tabular}

The main symptom of OS was local swelling, with 20 patients (95\%) experiencing swelling at the tumor site. Only four (19\%) reported pain at the time of admission. Six (29\%) experienced CNSrelated symptoms. Three (14\%) presented with symptoms similar to an infection. One OS was found coincidentally when the patient was examined by scintigraphy for another indication.

Fourteen tumors (67\%) were high-grade OSs and six (29\%) were of low-grade. It was not possible to determine the grade in one patient.

Surgical resection with curative intent was performed in 20 patients (95\%). In one patient the treatment consisted of palliative surgery and radiotherapy (50 Gy), and he died after 6 months. Six out of these 20 patients (30\%) underwent an ipsilateral neck dissection. The tumor removal was radical with clear margins in only five (25\%) of these 20 patients. Four (20\%) of tumor removals were intralesional; in one of these this was further completed with a re-resection to close margins. Altogether, 12 (60\%) of the resections with curative intent were performed with close margins.

Among the 20 patients treated with curative intent, preoperative chemotherapy (typically adriamycin - cisplatin) but no preoperative radiotherapy, was given to six patients (30\%). Postoperatively, 11 (55\%) of these 20 patients received either radiotherapy only $(n=6)$, chemotherapy only $(n=3)$, or both $(n=2)$, but two adjuvant therapies had to be stopped due to rapid tumor growth. Seven patients (35\%) received radiotherapy due to close or intralesional surgical margins, and the total dose varied between 50 Gy and 70 Gy. One patient did not receive any adjuvant therapy due to persistent tumor growth.

Tumor, treatment, and follow-up factors are presented in Table 3. Nine $(45 \%)$ of the 20 patients had a recurrence during follow-up. One recurrence was found in a patient who primarily had clear margins. On the other hand, seven patients with intralesional or close margins in their primary operation did not develop either recurrent or metastatic disease.

Most $(n=7)$ of the recurrences were seen during the first 6 months. In two patients in the whole series of 21 patients

Table 2

Five patients with previous radiotherapy for a head-and-neck malignancy.

\begin{tabular}{lll}
\hline Previous malignancy & Previous therapy & OS localization \\
\hline $\begin{array}{l}\text { SCC, mobile tongue } \\
\text { B-cell non-Hodgkin } \\
\text { lymphoma, orbit }\end{array}$ & $\begin{array}{l}\text { Surgery, radiotherapy } \\
\text { Radiotherapy, chemotherapy }\end{array}$ & $\begin{array}{l}\text { Mandible } \\
\text { Maxilla }\end{array}$ \\
$\begin{array}{l}\text { Oligodendroglioma } \\
\text { SCC, floor of mouth (FOM) }\end{array}$ & Radiotherapy & Radiotherapy \\
Oligodendroglioma & Radiotherapy & Mandible + FOM \\
\hline
\end{tabular}


Table 3

Tumor-, treatment-, and follow-up-related factors in 21 patients with CMF OS.

\begin{tabular}{|c|c|c|c|c|c|c|c|c|c|}
\hline Site & Grade & Preop.CT & Surgery & Margins & Postop. RT/CT & Recurr. & Metast. & Tx of rec. & Status at last $\mathrm{f} / \mathrm{u}$ \\
\hline \multicolumn{10}{|c|}{ Palliative intent of treatment $(n=1)$} \\
\hline Temp. & HG & & Enucleation & M & RT & $6-12 \mathrm{mo}$ & & & DOD \\
\hline \multicolumn{10}{|c|}{ Curative intent of treatment $(n=20)$} \\
\hline Mand. & HG & & $+($ Suppl. Sx $)$ & $\mathrm{I}>\mathrm{M}$ & & $<6 \mathrm{mo}$ & $<6 \mathrm{mo}$ & ND & DOD \\
\hline Max. & HG & + & + with ND & M & RT & & & & ANED \\
\hline Max. & HG & & $+($ Suppl. Sx) & I & & $<6 \mathrm{mo}$ & $<12 \mathrm{mo}$ & RT & DOD \\
\hline Front. & HG & + & + & M & & $<6 \mathrm{mo}$ & $<48 \mathrm{mo}$ & $\mathrm{Sx}+\mathrm{RT}$ & DOD \\
\hline Max. & HG & & + & M & $\mathrm{RT}+\mathrm{CT}$ & & & & ANED \\
\hline Max. & HG & & + & M & $\mathrm{CT}$ & & & & ANED \\
\hline Max. & HG & + & + & I & $\mathrm{CT}$ & $<6 \mathrm{mo}$ & & Sx & ANED \\
\hline Mand. & HG & + & + with ND & $\mathrm{M}$ & $\mathrm{RT}+\mathrm{CT}$ & $<12 \mathrm{mo}$ & $<18 \mathrm{mo}$ & $\mathrm{Sx}+\mathrm{CT}$ & DOD \\
\hline Max. & HG & + & + & Clear & RT & & & & ANED \\
\hline Front. & LG & & + & Clear & $\mathrm{CT}$ & & & & DOC \\
\hline Max. & $\mathrm{HG}$ & & + & $\mathrm{M}$ & & & & & DOD \\
\hline Mand. & $\mathrm{N} / \mathrm{A}$ & & Biopsy & $\mathrm{M}$ & & & & & ANED \\
\hline Mand. & LG & & + with ND & Clear & & $<54$ mo & & Sx & ANED \\
\hline Mand. & LG & & + with ND & M & RT & & & & ANED \\
\hline Mand. & HG & & + with ND & $\mathrm{M}$ & & $<6 \mathrm{mo}$ & $<6 \mathrm{mo}$ & & DOD \\
\hline Mand. & HG & & + with ND & Clear & & & & & DOC \\
\hline Front. & HG & & + & Clear & & & & & ANED \\
\hline Mand. & LG & & + & I & RT & $<6 \mathrm{mo}$ & & $\mathrm{CT}$ & DOD \\
\hline Zygom. & LG & + & + & $\mathrm{M}$ & RT & $<6$ mo & & Sx & DOD \\
\hline Max. & LG & & + & M & RT & & & & ANED \\
\hline
\end{tabular}

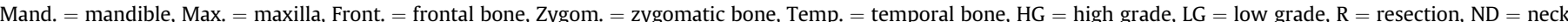

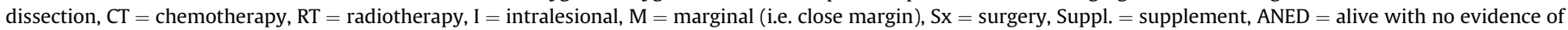
disease, $\mathrm{DOD}=$ dead of disease, $\mathrm{DOC}=$ dead of other cause, $\mathrm{f} / \mathrm{u}=$ follow-up.

recurrence was found between 6 and 12 months. One recurrence was detected 4 years after the primary operation.

Five patients (25\%) developed postoperative metastatic disease; earlier recurrent disease had already been detected in these cases. Three out of those 11 patients who had been operated on with curative intent, and had received postoperative oncological therapy, died of the disease during follow-up, and one died due to another cause.

Ten patients (50\%) are alive with no evidence of disease after their follow-up. Eight patients (40\%) died due to OS, with an average survival time of 1.3 years (median 0.9 ; range $0.3-3.8$ ). Two patients (10\%) died due to another cause.

\section{Discussion}

Craniomaxillofacial OS is extremely rare, and large studies aiming at investigating predictive factors for treatment outcome are sparse. In Finland, with a current population of 5.5 million, there were only 21 new patients with a histologically verified OS in the CMF area during the 17-year period from 1992 to 2009. We reviewed the management and long-term outcomes for this nationwide series in order to update our current treatment protocol.

CMF OS accounts for 10-15\% of all osteosarcomas (Jasnau et al., 2008; Sampo et al., 2011). The total number of all osteosarcomas in Finland during a 14-year period from 1991 to 2005 was 144, and this series included four (3\%) CMF OS patients (Sampo et al., 2011). Our study reports an annual occurrence of 0.02 new cases per 100,000 inhabitants for CMF OS in Finland.

The average age at the onset of CMF OS is typically higher than that for the extremities (Lee et al., 2015). During the 5-year period from 2001 to 2005 the average age of all OS patients in Finland was 31 years (Sampo et al., 2011). This is almost 10 years lower than the average age (39.6 years) in the our study. Although the mean age of all OS patients in Finland seems to be increasing, the average age of CMF OS patients is clearly higher. Similarly, Fernandez et al. reported an average age of 41 years (range 14-51) in a series of 16 patients with maxillofacial OS (Fernandes et al., 2007). In a series of
541 patients diagnosed with osteosarcoma of the jaws, the mean age was 41.3 years (Lee et al., 2015).

The majority (67\%) of the patients in our study were male. This differs slightly from some other published reports that had a female preponderance (Lee et al., 2015; Chidzonga and Mahomva, 2007; Ferdandez et al., 2007; Jasnau et al., 2008). On the other hand, in the studies by Thiele et al. and Laskar et al. the gender ratio was similar that in our study (Thiele et al., 2008; Laskar et al., 2008). Interestingly, the male-to-female ratio for all OSs in Finland two decades ago was 1.9 , but seems to be reversing as it was only 0.6 three decades ago (Sampo et al., 2011). This change has not been observed in CMF OS.

Most of the OSs in the CMF region arise in either mandibular or maxillary bone. Twenty-four per cent in our study were located in facial and skull bones other than the maxilla or mandible. This incidence was lower than presented by other studies (Jasnau et al., 2008). The main symptom of CMF OS in our series was local swelling, with over $90 \%$ of our patients presenting with this sign. Only four (19\%) of the 21 patients experienced pain and fewer than $10 \%$ of the patients reported loss of skin sensation. These findings are supported by other studies (Ketabchi et al., 2011; Chidzonga and Mahomva, 2007).

Five (23\%) of our 21 patients had previously been treated for malignant disease an average of 9 years earlier. More importantly, they had all received radiotherapy in the site of their CMF OS. Earlier studies have stated that the prevalence of secondary OS is low in general, and is typically induced by radiotherapy (Valenti et al., 2005; Wood et al., 2015; Giannini et al., 2018).

Surgery is the main treatment modality for CMF OS (Kämmerer et al., 2012; Jasnau et al., 2008; Ketabchi et al., 2011; Krishnamurthy and Palaniappan, 2018). In our study, 95\% of the patients were operated on with curative intent. Neck dissection was performed in $30 \%$ of the patients; most often this was performed to facilitate microvascular reconstructive surgery. The main difficulty associated with CMF OS surgery is achieving disease free margins. Clear margins were achieved only in $25 \%$ (5 out of 20 ) of the cases with curative treatment intent in our series, although this was the intention in all of those patients. In almost two thirds (60\%) of these 
20 cases the resection resulted in close margins, and in $20 \%$ the initial resection was intralesional. There are only a few studies with documented histological radicality of the resection. In parallel with our study, the COSS study reported an intralesional resection rate of 26\% (Jasnau et al., 2008).

There are not many reports on the effectiveness of preoperative chemotherapy in CMF OS (Mardinger et al., 2001). Six patients in our series received preoperative chemotherapy. This had to be stopped for two of them due to rapid and persistent tumor growth. The COSS study group reported poor response to chemotherapy (a good response being $<10 \%$ viable tumor) in $66 \%$ of patients in a subgroup of maxillofacial OS patients $(n=16)$ who had received preoperative chemotherapy (Jasnau et al., 2008). This finding is consistent with our study, although the numbers are very low in both studies. This may indicate that, in contrast to preoperative chemotherapy in OS of extremities, preoperative chemotherapy in maxillofacial OS is not as effective. This might be due to the fact that, embryologically, facial bones are different from long bones. Additionally, the vascularity around the facial region is richer than in the extremities, which might have an effect on the recovery of the tumor cells after chemotherapy.

Free resection margins appear essential in preventing development of local recurrence and metastasis. All three patients with an intralesional or suspected intralesional resection developed local recurrence in our study. On the other hand, only one out of five patients with good resection margins developed recurrence, and that was more than 4 years after the primary surgery. In that case the OS was low grade and, after salvage surgery, the patient continues to be disease free after a 21-year follow-up. Therefore, in our study, aggressive surgery with clear free margins seemed to decrease the risk of recurrence and metastasis, which has also been suggested by others (Ketabchi et al., 2011; Laskar et al., 2008; Chen et al., 2016). However, six patients with intralesional or close margins in their primary operation did not develop either recurrent or metastatic disease, which remains an inconclusive finding of our study. This observation may be indicative for mandatory postoperative therapy instead of preoperative adjuvant treatment. This approach would also shorten the delay for radical surgery. Further and larger studies are needed to evaluate the value of the this proposed management approach.

Half of the patients in our series survived with no evidence of OS at the last follow-up visit, while two patients died of another cause, which corresponds well with other studies (Kämmerer et al., 2012; Laskar et al., 2008). Eight (53\%) of the 15 patients with intralesional or close margins died due to OS.

It is interesting that all patients with metastatic spread during the postoperative follow-up also had local recurrence. All those patients died of their disease in spite of several treatment modalities. Therefore, based on the our results and those of others, it seems that the usefulness of salvage surgery for patients with CMF OS recurrence and metastasis should be carefully and individually considered (Lee et al., 2015; Jasnau et al., 2008).

\section{Conclusion}

Postoperative radiotherapy and/or chemotherapy was shown to be fairly effective in this CMF OS patient population, with only $27 \%$ (3/11) of these patients dying of their disease during follow-up. This is notable because postoperative therapy was indicated and administered in cases with intralesional or close margins. This finding is supported by other studies (Thiele et al., 2008; Kämmerer et al., 2012; Jasnau et al., 2008; Ketabchi et al., 2011).

\section{Declarations of interest \\ None.}

\section{Acknowledgements}

This study was supported by the Helsinki University Hospital Research Fund.

\section{Appendix A. Supplementary data}

Supplementary data to this article can be found online at https://doi.org/10.1016/j.jcms.2019.03.020.

\section{References}

August M, Magennis P, Dewitt D: Osteogenic sarcoma of the jaws: factors influencing prognosis. Int J Oral Maxillofac Surg 26(3): 198-204, 1997

Chen Y, Shen Q, Gokavarapu S, Lin C, Yahiya, Cao W, et al: Osteosarcoma of head and neck: a retrospective study on prognostic factors from a single institute database. Oral Oncol 58: 1-7, 2016 Jul

Chidzonga MM, Mahomva L: Sarcomas of the oral and maxillofacial region: a review of 88 cases in Zimbabwe. Br J Oral Maxillofac Surg 45(4): 317-318, 2007 Jun

Fernandes R, Nikitakis NG, Pazoki A, Ord RA: Osteogenic sarcoma of the jaw: a 10year experience. J Oral Maxillofac Surg 65(7): 1286-1291, 2007 Jul

Giannini L, Incandela F, Fiore M, Gronchi A, Stacchiotti S, Sangalli C, et al: Radiationinduced sarcoma of the head and neck: a review of the literature. Front Oncol 8 : 449, 2018 Oct

Guadagnolo B, Zagars G, Raymond A, Benjamin R, Sturgis E: Osteosarcoma of the jaw/craniofacial region. Outcomes after multimodality treatment. Cancer 115: 3262-3270, 2009

Jasnau S, Meyer U, Potratz J, Jundt G, Kevric M, Joos UK, et al: Craniofacial osteosarcoma experience of the cooperative German-Austrian-Swiss osteosarcoma study group. Oral Oncol 44(3): 286-294, $2008 \mathrm{Mar}$

Kämmerer PW, Shabazfar N, Vorkhshori Makoie N, Moergel M, Al-Nawas B: Clinical therapeutic and prognostic features of osteosarcoma of the jaws - experience of 36 cases. J Craniomaxillofac Surg 40(6): 541-548, 2012

Ketabchi A, Kalavrezos N, Newman L: Sarcomas of the head and neck: a 10-year retrospective of 25 patients to evaluate treatment modalities, function and survival. Br J Oral Maxillofac Surg 49(2): 116-120, 2011 Mar

Krishnamurthy A, Palaniappan R: Osteosarcomas of the head and neck region: a case series with a review of literature. J Maxillofac Oral Surg 17(1): 38-43, 2018

Laskar S, Basu A, Muckaden MA, D'Cruz A, Pai S, Jambhekar N, et al: Osteosarcoma of the head and neck region: lessons learned from a single-institution experience of 50 patients. Head Neck 30(8): 1020-1026, 2008 Aug

Lee RJ, Arshi A, Schwartz HC, Christensen RE: Characteristics and prognostic factors of osteosarcoma of the jaws: a retrospective cohort study. JAMA Otolaryngol Head Neck Surg 141(5): 470-477, 2015 May 1

Mardinger O, Givol N, Talmi YP, Taicher S: Osteosarcoma of the the jaw. The chaim Sheba Medical center experience. Oral Surg Oral Med Oral Pathol Oral Radio Endod 91(4): 445-451, 2001 Apr

Sampo MM, Tarkkanen M, Kivioja AH, Taskinen MH, Sankila R, Böhling TO: Incidence, epidemiology and treatment results of osteosarcoma in Finland - a nationwide population-based study. Acta Oncol 50(8): 1206-1214, 2011 Nov

Sumida T, Otawa N, Kamata YU, Yamada T, Uchida K, Nakano H, et al: A clinical investigation of oral sarcomas at multi-institutions over the past 30 years. Anticancer Res 35(8): 4551-4555, 2015 Aug

Thiele OC, Freier K, Bacon C, Egerer G, Hofele CM: Interdisciplinary combined treatment of craniofacial osteosarcoma with neoadjuvant and adjuvant chemotherapy and excision of the tumour: a retrospective study. $\mathrm{Br} \mathrm{J}$ Ora Maxillofac Surg 46(7): 533-536, 2008 Oct

Valentí V, López-Pousa A, Gonzalez Y, Farré N: Radiation-induced mandibular osteogenic sarcoma: report of a case and review of the literature. J Craniofac Surg 16(3): 452-456, 2005 May

Wood J, Ver Halen J, Samant S, Florendo N: Radiation-induced sarcoma masquerading as osteoradionecrosis: case report and literature review. J Laryngol Otol 129(3): 279-282, 2015 Mar

Yamaguchi S, Nagasawa H, Suzuki T, Fujii E, Iwaki H, Takagi M, et al: Sarcomas of the oral and maxillofacial region: a review of 32 cases in 25 years. Clin Oral Invest 8: 52-55, 2004 